\title{
Design and Evaluation of Chitra Swab Collection Booths for Health Professionals in COVID-19 Pandemic
}

\author{
Saurabh Sudhakaran Nair ${ }^{1}$ Arvind Kumar Prajapati ${ }^{1}$ [D $\cdot$ Ramesh Babu Venkatesan ${ }^{1}$. \\ Muraleedharan Chirathodi Vayalappil ${ }^{1} \cdot$ Asha Kishore $^{2}$
}

Received: 10 July 2020 / Accepted: 24 August 2020 / Published online: 31 August 2020

C Indian National Academy of Engineering 2020

\begin{abstract}
The 2019 novel coronavirus (SARS-CoV-2), officially named as COVID-19 by the WHO, has spread to more than 180 countries and the confirmed coronavirus cases have reached around 10 million with 0.6 million deaths by end of June 2020. Moreover, there is no sign of a sustained decline in any country till date. Continuous rise of positive cases has instilled fear in people, society and even health professionals. According to WHO's daily situation report, 22,073 COVID-19 cases of healthcare professionals have been reported to the WHO as of Wednesday, 8 April 2020 by Jin (Mil Med Res 7:24, 2020). Infection to health professionals is a serious concern not only because they are a valuable frontline worker but also because of the risk of spread to co-workers and non-Covid patients. This project was undertaken to develop a solution to minimize the chance of infection to the health care professionals by providing them isolation from a potential source of Covid-19 and similar highly contagious diseases. The two models of Chitra swab collection booth were developed to: (1) protect health professionals from the risk of infection (2) to provide technical know-how to manufacturers to produce booths using locally available materials while meeting international regulations and (3) reduce the consumption of personal protective equipment. The prototypes developed were tested for safety and efficacy in accordance with the guidelines of the Centers for Disease Control and Prevention, Atlanta, USA. The device received the registration for commercialisation from the Central Drugs Control Standard Organization, Ministry of Health, Government of India, as a non-notified medical device.
\end{abstract}

Keywords Swab collection booth $\cdot$ SARS-Cov-2 $\cdot$ COVID-19 $\cdot$ HEPA filter $\cdot$ UV-C $\cdot$ Healthcare professional

\section{Introduction}

In December 2019, a cluster of cases of respiratory tract infections caused by a novel coronavirus was reported in Wuhan, the heart of Hubei province of China (Huang et al. 2019). Soon after, several cases of COVID-19 outbreaks were reported in various parts of the world, which led to the world health organization declaring the escalating situation as a global Pandemic in March 2020. The rapid increase in number of cases of Covid-19 is of grave concern to people

Arvind Kumar Prajapati

arvind@sctimst.ac.in

1 Department of Medical Devices Engineering, Sree Chitra Tirunal Institute for Medical Sciences and Technology, Thiruvananthapuram, India

2 Department of Neurology, Sree Chitra Tirunal Institute for Medical Sciences and Technology, Thiruvananthapuram, India and health care professionals. The reports from medical staff from various hospitals describe the physical and mental exhaustion they suffer, their inability to decide the order of treatment to be provided because of the overwhelming number of cases, the fear of losing their patients and family members and above all the risk of getting infected themselves to a potentially fatal disease.

Providing a safe and effective device that provides a physical barrier to doctors while examining their patients or while collecting samples from their patients would be the first line of protection from contracting the virus. The second would be to maintain the place of examination or sample collection free of the virus. The physical barrier can be achieved by building a telephone booth-like enclosure using readily available aluminium composite panels or extruded PVC panels on aluminium extrusions frame. A virus-free environment in the booth could be achieved by maintaining negative pressure in the patient chamber and treating the viral load shed by the patient using UV-C lights. 
There are numerous methods developed in the past for inactivation of microorganisms of which Ultraviolet C (UVC) germicidal disinfection method uses UV-C radiation to disinfect microorganisms (Hijnen et al. 2006). Microorganisms such as bacteria are unicellular and contain deoxyribonucleic acid (DNA) whereas viruses are smaller only enveloped by a protein coat (CIE 2003). Viruses contain one or more molecules of DNA or Ribonucleic acid (RNA). UV-C exposure results in the destruction of nucleic acid present in microorganisms through induction of hydrates, uracil and thymine dimers. The absorption of UV-C by the RNA or DNA of a microorganism leads to its photochemical destruction. This prevents the replication of the microorganism and its subsequent inactivation (Neelakandan et al. 2020).

This device was developed to achieve the following; (1) reduce the risk of infection to health professionals (2) generate the technical know-how to produce device using local resources with minimal material movement (3) provide a design based on already well-established scientific evidence so that biological validation would be minimised under the lockdown conditions of Covid-19 (4) reduce PPE requirements.

Booths based on similar rationale have been previously developed and reported (Kim and Lee 2020; Joshi 2020). The Korean Walk-Through Screening Center (Kim and Lee 2020) focuses on generating an efficient layout to screen a large number of patients, which is meant for use in large hospitals and screening centres. The innovative portable sample kiosk (Joshi 2020) is a device with the facilities for automated disinfection after every use, and this feature is likely to make it costlier. However, Chitra swab collection booths are conceived as cost-effective standalone devices. These can be deployed in a hospital or public health centres either as a standalone module or as a cluster. Further, the UV-C based disinfection of exhaust air along with HEPA filter provides two-stage disinfection before the air is let out to the environment.

\section{Methods}

\section{Chitra Swab Collection Booths}

Figure 1 shows the component details and Fig. 2 shows details of airflow direction in Chitra Swab collection booth. The design is a dual-compartment enclosure that provides a physical barrier for health professional during swab collection (Nasopharyngeal swab/Oropharyngeal swab) from potentially infected persons. The bottom compartment is for the patient or a suspected case and has $1.8 \mathrm{~m}^{2}$ area and $2 \mathrm{~m}$ height, maintained under negative pressure to minimize the spread of the virus to the outside environment. This compartment is equipped with longsleeved gloves to be worn by health worker while reaching the patient for the collection of swab samples. Neoprene or latex gloves can be used and will need periodic replacement. These gloves are attached to a cylindrical tube provided on the bottom compartment using hose clips which can be tightened onto the cylindrical tube providing airtight seal. The patient entry gate faces the glove side of the compartment. A wireless portable interphone is installed for easy communication. All consumables necessary for
Fig. 1 Schematic of Chitra Swab collection booth with components details

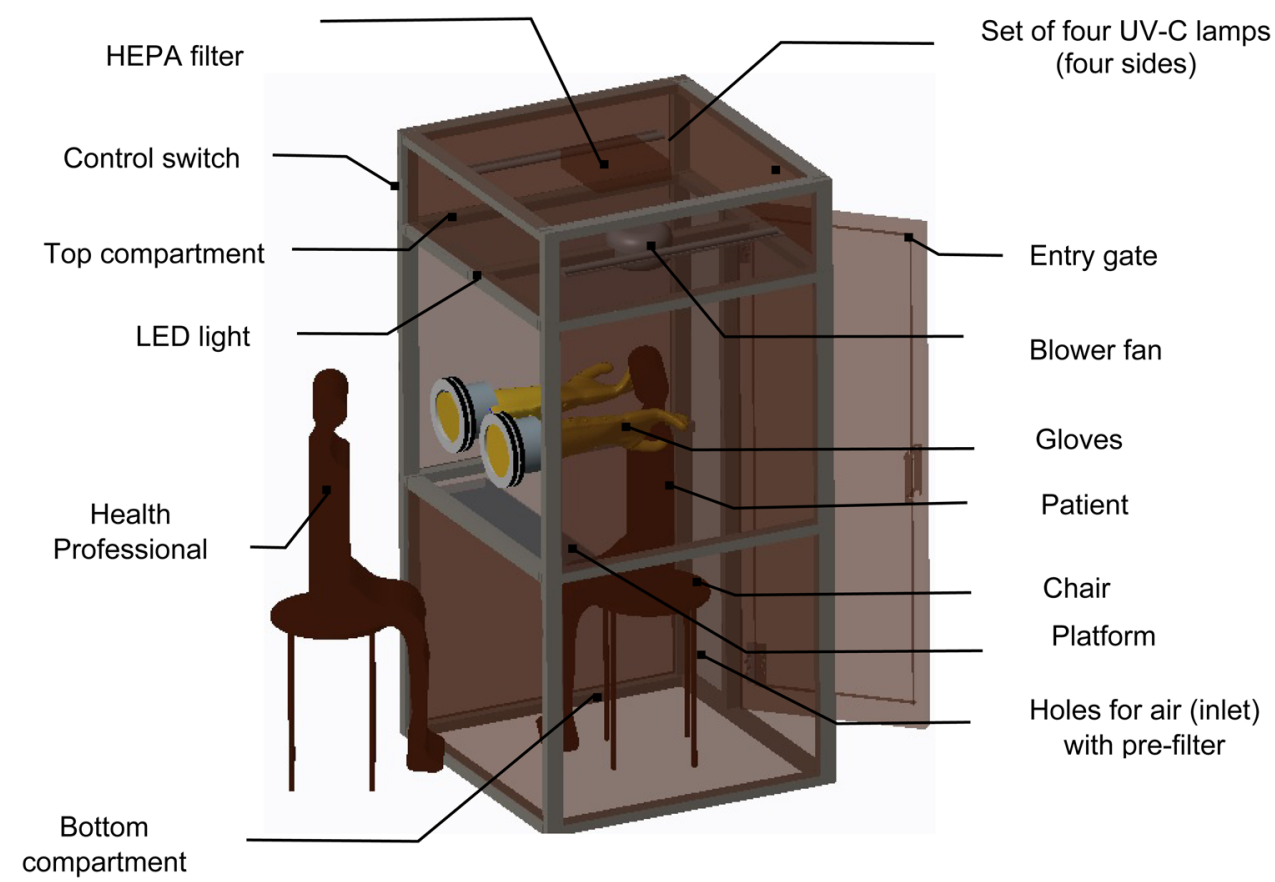




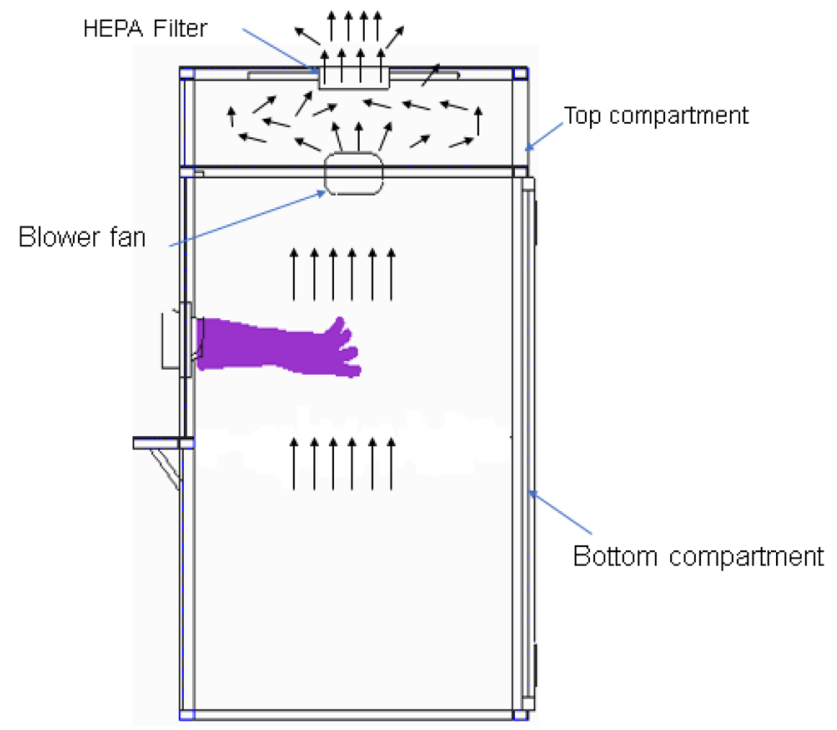

Fig. 2 Schematic showing the airflow in Chitra Swab collection booth

swab collection are provided in the bottom compartment. Additionally, Health professional and patient chamber can be equipped with customized seating arrangements (e.g. chairs and tables) to improve the working conditions.

The booth is fabricated using conventional aluminium extrusions $(60 \mathrm{~mm} \times 40 \mathrm{~mm}$ nominal cross-section) and either aluminium composite panels or extruded PVC panels (5-10 $\mathrm{mm}$ nominal thickness). The transparency in the gloves area is achieved using toughened and annealed glass with 6-10 mm nominal thickness. A $230 \mathrm{~V}, 8 \mathrm{~W}$ LED lamp is provided for interior lighting.

The air from the bottom compartment is drawn into the top compartment as shown in Fig. 2, with $1.8 \mathrm{~m}^{2}$ area and $0.4 \mathrm{~m}$ height. The top compartment has provision to disinfect air before being let out. To enable this, the top compartment made of aluminium composite or extruded PVC panels is equipped with a high-efficiency particulate air filter (HEPA) (0.3-micron nominal cut off) with 100-150 $\mathrm{m}^{3} / \mathrm{h}$ airflow capacity against $50 \mathrm{~Pa}$ differential pressure, a set of 4 UV-C lamps ( $254 \mathrm{~nm}, 30 \mathrm{~W}$ each) and a conventional blower $\left(100-150 \mathrm{~m}^{3} / \mathrm{h}\right)$ matched to the HEPA filter to generate the pressure differential in the chamber. Exhaust air from the bottom compartment enters the top compartment and gets flushed out via the HEPA filter as shown in Fig. 2, which traps fine viral matter. The UV lamps disinfect the trapped as well as suspended viral load. A sample volume of air in the top compartment gets UV exposure for a minimum of $20 \mathrm{~s}$ before it reaches to HEPA filter. This ensures that the major portion of suspended viral load is disinfected before it reaches the filter and the HEPA filter provides the second line of defence.
Before the device is used, booth including the gloves and door handles shall be disinfected using an appropriate chemical disinfectant (Sodium Hypochlorite solution or mild detergents). Disinfection must be repeated after every patient exits. During the period of disuse, the booth can be switched off. Users need to ensure that the device is switched on at least $15 \mathrm{~min}$ before the next use.

In a second step, the model was further refined as a dualchamber swab collection booth, to provide protection and prevent cross infection between the health professional and an asymptomatic person who is sampled. The Chitra dualchamber booth has a chamber for the health professional and the second chamber for the patient or an asymptomatic person. The two chambers are separated by a transparent glass barrier on which, a pair of long-sleeved gloves are mounted. The patient chamber design details are the same as Chitra swab collection booth.

As shown in Fig. 3, the health professional's chamber has a single door entry and is equipped with an LED lamp and a compact wall-mounted fan. The health professional's chamber has $1.8 \mathrm{~m}^{2}$ area and $2 \mathrm{~m}$ height. The gate of the health professional's chamber is opposite to patient entry gate. Additionally, a wireless portable interphone is installed for ease of communication. All consumables necessary for swab collection are provided in patient chamber.

The bill of materials for the single-chamber booth is estimated at about ₹ $75 \mathrm{~K}$ and that of dual chamber at ₹ $115 \mathrm{~K}$.

\section{Safety and Efficacy Evaluation}

\section{Negative Pressure Inside Bottom Compartment of Booth}

A M2 Series Smart Manometer (M/s Meriam, USA) was used for pressure differential measurement. One limb of the manometer was held inside the booth while the other limb was routed outside the booth via a small hole made through a plastic sheet covering glove-port opening. Measurements were taken with the booth door shut and blower fan turned on. It was ensured that small air gaps at the roof edges of the bottom chamber were sealed using silicone to avoid leakage and backflow of exhaust air from the top chamber. The stability of the negative pressure in the bottom compartment was ascertained by four replicated measurements.

\section{Number of Air-Changes within the Booth}

A DIGISENSE hotwire anemometer (M/s Cole-Parmer, USA) was used to measure the velocity of air exiting the HEPA filter. The telescoping hot-wire probe was placed at multiple locations above the HEPA filter and respective velocity was measured. A rectangular wooden box of the same size as HEPA filter was placed on top of the filter and small air gaps at the interface were sealed using silicone to avoid leakage and side 
Fig. 3 Schematic showing Chitra dual-chamber swab collection booth

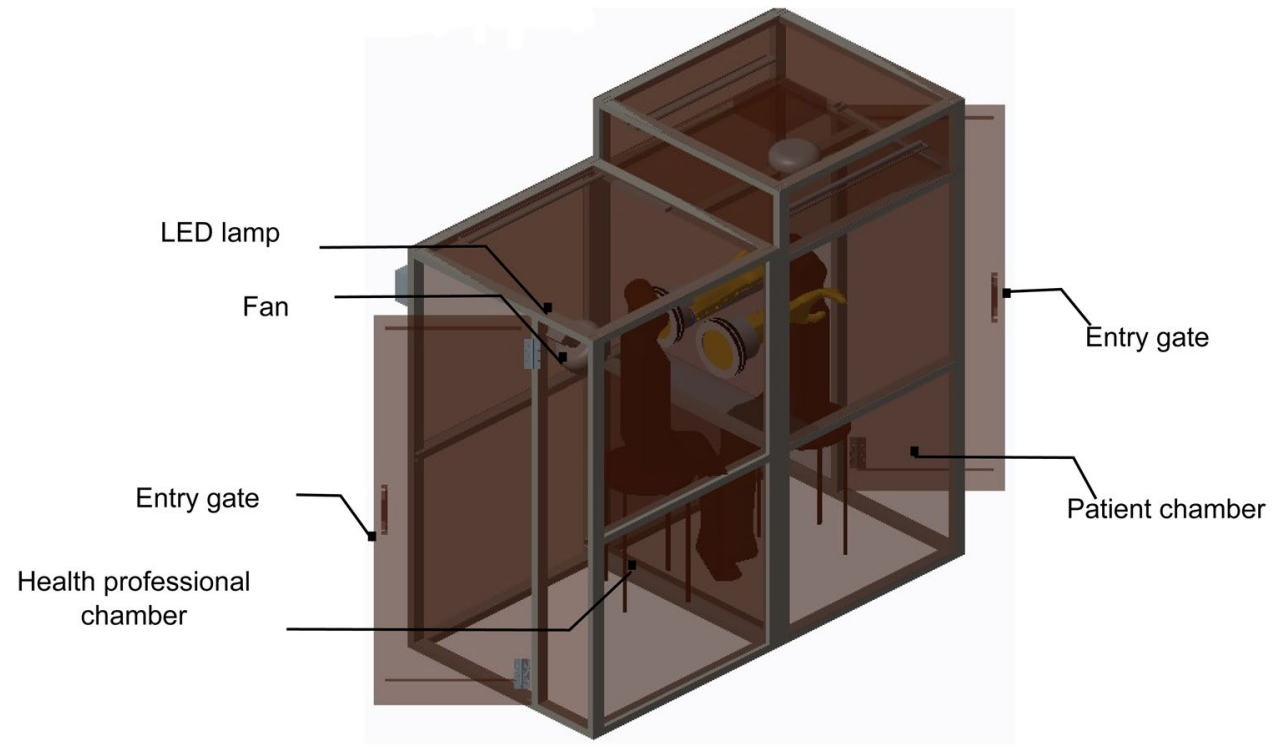

flow of exhaust air during measurements. The average air velocity was used for estimating airflow through the HEPA filter and was translated to a number of air changes per hour.

\section{UV Power Density}

A set of four $30 \mathrm{~W}, 254 \mathrm{~nm}$ UV lamps were fit inside the top compartment (four sides) of the Chitra devices to disinfect the air in the top compartment. The $254 \mathrm{~nm}$ component of UV dominated the spectral power distribution of the lamp. Measurements were performed at several locations near the HEPA filter. An 843-R (M/s Newport, USA) laser power meter with a thermopile detector (919P-003-10 (M/s Newport, USA)) was used for UV power measurements.

\section{Results}

\section{Negative Pressure Inside Bottom Compartment of Booth}

The measurements obtained using Smart Manometer gives average negative pressure differential of $12 \pm 1 \mathrm{~Pa}$ (Fig. 4). Thus, the exhaust system used in the Chitra devices maintains bottom compartment at a negative pressure differential of $12 \mathrm{~Pa}$, which meets the guidelines issued by Centers for Disease Control and Prevention (Centers for Disease Control and Prevention 2003).

\section{Number of Air-Changes within the Booth}

The exhaust air velocity above the HEPA filter was measured to be $0.4 \pm 0.2 \mathrm{~m} / \mathrm{s}$ (Fig. 5). The flow rate of exhaust air exiting the filter was calculated as follows.

Average velocity, $v=0.4 \pm 0.2 \mathrm{~m} / \mathrm{s}$.

\section{Negative Differential Pressure vs Time}

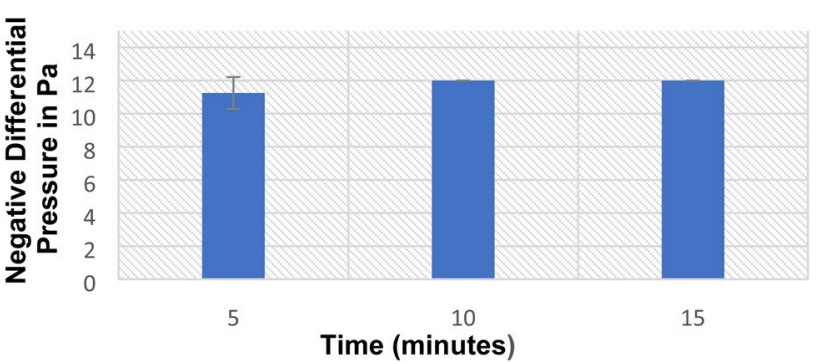

Fig. 4 Measured negative differential pressure values in a booth at different time

HEPA filter area, $A=0.30 * 0.30 \mathrm{~m}^{2}=0.09 \mathrm{~m}^{2}$.

Flow rate of exhaust air exiting the filter $=A^{*} v^{*} 3600=129.6 \mathrm{~m}^{3} / \mathrm{h}$, this translates to 30 airchanges per hour, with a chamber volume of $4.32 \mathrm{~m}^{3}$, which meets the guidelines issued by Centers for Disease Control and Prevention (Centers for Disease Control and Prevention 2003).

\section{UV Power Density}

Figure 6 shows the UV power density measurements in the proximity of the HEPA filter in the four corners inside the top compartment. The average power density observed was $0.24 \pm 0.07 \mathrm{~mW} / \mathrm{cm}^{2}$. The International Ultraviolet Association suggests UV dose of $40 \mathrm{~mJ} / \mathrm{cm}^{2}$ for disinfection of viruses on flat, ideal surfaces (International Ultraviolet Association 2020). This dose is accumulated in within 3 min at the said power density with the configuration of lamps used in the swab collection booth. 


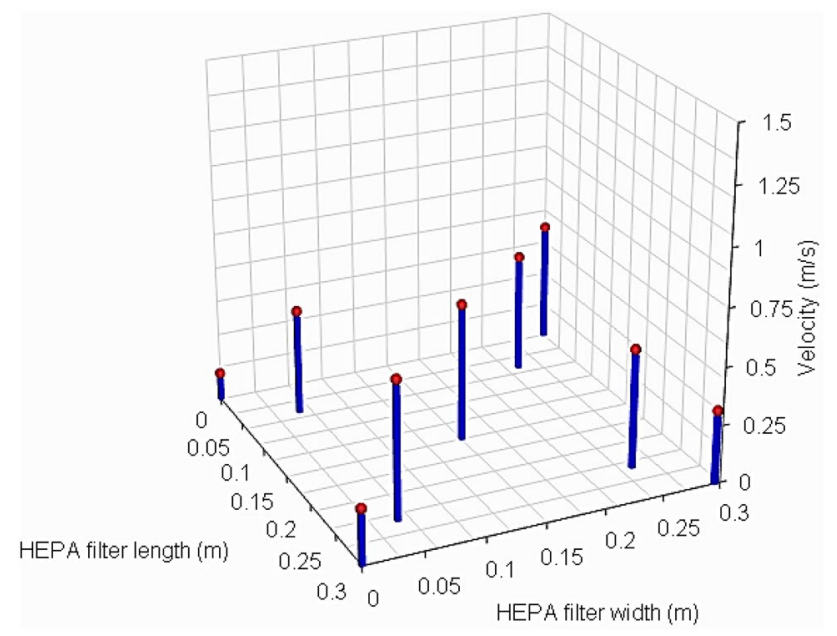

Fig. 5 Measured velocity at various locations on the top surface of HEPA filter

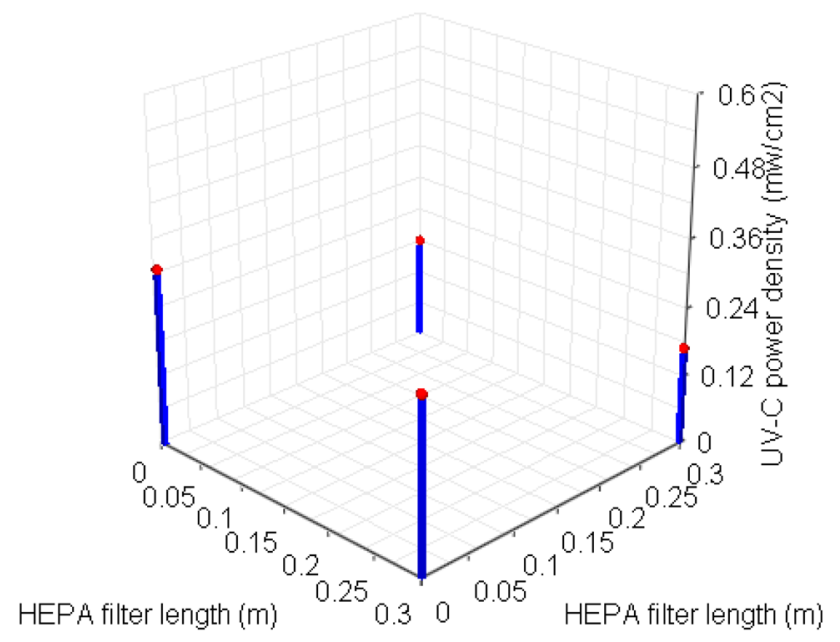

Fig. 6 Measured UV-C power density at various locations on the bottom surface of HEPA filter

\section{Discussion}

The designs of both single and dual-chamber booths aim to protect health professional from the risk of infection during swab collection during the current Covid-19 pandemic. The concept of providing negative pressure in the patient chamber was inspired by the biosafety cabinet and is one of the important safety features in both the devices. The devices produced negative pressure differential of $12 \pm 1 \mathrm{~Pa}$ and 30 air changes per hour, which provides a safety margin of 4 in the case of negative pressure and 2.5 in the case air changes (based on the threshold levels $>2.5 \mathrm{~Pa}$ and $>12$ air changes per hours in air born infection control isolation) respectively and as set by CDC guidelines (Centers for Disease Control and Prevention 2003).

The International Ultraviolet Association, recommends UV dose (power density multiplied by the time in seconds) of at least $40 \mathrm{~mJ} / \mathrm{cm}^{2}$ to make viruses non-functional on surfaces (perfectly flat and ideal surfaces) for a device placed at a fixed distance and is used to disinfect a surface (International Ultraviolet Association 2020). Both devices produced an average power density of $0.24 \pm 0.07 \mathrm{~mW} / \mathrm{cm}^{2}$ on the HEPA filter, which accumulated to $43 \mathrm{~mJ} / \mathrm{cm}^{2}$ in within 3 min. Additionally, the UV-C lamps were kept continuously on in top compartment throughout the session. This provides an additional margin of safety to the devices.

The use of such devices can reduce the need of a frequent change of PPEs during swab collection, thus reducing the consumption of PPEs as well as the number of laborious donning and doffing procedures the health care professionals have to undergo.

The Chitra devices received registration from Central Drugs Control Standard Organization (CDSCO), Ministry of Health, Government of India, as a non-notified medical device.

Acknowledgements We greatly appreciate the contributions of the staff members from the department of Medical Devices Engineering, Sree Chitra Tirunal Institute for Medical Sciences and Technology, Trivandrum. The technology was transferred to multiple industry partners (M/s. HLL Lifecare Limited, Thiruvananthapuram, M/s. HMG (India), Mumbai, M/s. JADRO Steel LLP, Panchla, Howrah, Kolkatta, M/s. Sivapriya Exim Pvt.Ltd, Annanagar, Chennai, M/s. TVS Supply Chain Solution Limited, Indiranagar, Bengaluru, M/s. Kanjikode Industries Forum, Kerala, through the expression of interest. The presented work was funded by the Technical Research Centre (TRC) for Biomedical Devices, Department of Science and Technology (DST), Govt. of India. We also thank M/s. Fly Tech Industries, Thiruvananthapuram for initial prototyping and technical support.

\section{References}

Centers for Disease Control and Prevention (2003) Guidelines for Environmental Infection Control in Healthcare Facilities. www. cdc.gov

Hijnen WA, Beerendonk EF, Medema GJ (2006) Inactivation credit of UV radiation for viruses, bacteria and protozoan (oo) cysts in water: a review. Water Res 40(1):3-22

Huang C, Wang Y, Li X et al (2020) Clinical features of patients infected with 2019 novel coronavirus in Wuhan China. Lancet 395(10223):497-506

International Ultraviolet Association (2020) Advice for selection and operation of equipment for UV disinfection of air and surfaces. www.iuva.org

Jin Y, Huang Q, Wang Y et al (2020) Perceived infection transmission routes, infection control practices, psychosocial changes, and management of COVID-19 infected healthcare workers in a tertiary acute care hospital in Wuhan: a cross-sectional survey. Mil Med Res 7:24. https://doi.org/10.1186/s40779-020-00254-8

Joshi JR (2020) COVSACK: an innovative portable isolated and safe COVID-19 sample collection kiosk with automatic disinfection. 
Trans Indian Natl Acad Eng. https://doi.org/10.1007/s41403-02000139-1

Kim SI, Lee JY (2020) Walk-through screening center for COVID19: an accessible and efficient screening system in a pandemic situation. J Korean Med Sci 35(15):e154. https://doi.org/10.3346/ jkms.2020.35.e154

Neelakandan SN, Sukesan A, Jerard J, Uthaman VP, Vasudevan VD, Sarojini Amma P, Nandkumar MA, Vayalappil MC (2020) Chitra
ultraviolet-C-based facemask disposal bin. Trans Indian Natl Acad Eng. https://doi.org/10.1007/s41403-020-00136-4

Publisher's Note Springer Nature remains neutral with regard to jurisdictional claims in published maps and institutional affiliations. 